\title{
A NOTE ON AUTOMORPHIC FORMS OF WEIGHT ONE AND WEIGHT THREE
}

BY

PETER F. STILLER ${ }^{1}$

\begin{abstract}
In this paper the author develops an interesting relationship between classical automorphic forms of weights one and three, and the solutions of certain second order differential equations related to elliptic (modular) surfaces. In particular for a cusp form of weight three, it is shown that the special values of the associated Dirichlet series can be determined from the periods of an inhomogeneous differential equation, or what is the same thing, the monodromy of an associated third order differential equation. Explicit examples are provided for principal congruence subgroups $\Gamma(N)$ with $N \equiv 0 \bmod 4$.
\end{abstract}

1. Forms of weight one and differential equations. In this paper we wish to explain an interesting relationship between classical automorphic forms of weights one and three, and the solutions of certain second order differential equations related to elliptic surfaces.

Let $\mathfrak{h}=\{z \in \mathbf{C}: \operatorname{Im} z>0\}$ denote the complex upper half-plane. We wish to consider automorphic forms of weight $k$ with respect to a Fuchsian group of the first kind $\Gamma \subset \mathrm{SL}_{2}(\mathbf{R})$ so that $\mathfrak{h} * / \Gamma=X$ is a compact Riemann surface, where $\mathfrak{h} *$ is $\mathfrak{h}$ union the cusps of $\Gamma$ (see Shimura [17]). Recall that an automorphic form of weight $k$ with respect to $\Gamma$ is a meromorphic function $f$ on $\mathfrak{h}$ such that

$$
f(\gamma z)=(c z+d)^{k} f(z), \quad z \in \mathfrak{h},
$$

where $\gamma=\left(\begin{array}{ll}a & b \\ c & d\end{array}\right) \in \Gamma \subset \mathrm{SL}_{2}(\mathbf{R})$ acts by linear fractional transformation on $\mathfrak{h}$. Moreover, $f$ must be meromorphic at the cusps (see Shimura [17]).

Suppose we are given such a meromorphic function $f$ of weight $k=1$. (We limit ourselves to this case, but clearly the construction below carries through to higher weight.) Consider the function $g(z)=z f(z)$. This function transforms as

$$
g(\gamma z)=(a z+b) f(z) .
$$

If we select a Zariski open subset $X_{0} \subset X$ appropriately, we can regard $g(x), f(x)$ as a pair of multivalued holomorphic functions on $X_{0}$. Moreover, if we fix a base point

Received by the editors December 23, 1982 and, in revised form, August 31, 1984.

1980 Mathematics Subject Classification. Primary 10Dxx, 14Dxx.

Key words and phrases. Elliptic surface, automorphic form, $q$-expansion, $K$-equations, Gauss-Manin connection, monodromy.

${ }^{1}$ The author wishes to express his thanks to the Sonderforschungsbereich, Universität Bonn for its financial support during the preparation of this paper. 
$x_{0} \in X_{0}$, then for a path $\rho \in \pi_{1}\left(X_{0}, x_{0}\right)$ we have that $g$ and $f$ analytically continue around $\rho$ to

$$
a g(x)+b f(x), \quad c g(x)+d f(x), \quad\left(\begin{array}{ll}
a & b \\
c & d
\end{array}\right) \in \Gamma,
$$

respectively, i.e. $\left(\begin{array}{l}g \\ f\end{array}\right) \rightarrow\left(\begin{array}{ll}a & b \\ c & d\end{array}\right)\left(\begin{array}{l}g \\ f\end{array}\right)$. Denote by $K(X)$ the function field of $X$ (which is isomorphic to the field of modular functions on $\mathfrak{h}$ with respect to $\Gamma$ ) and select $x \in K(X)$ nonconstant. Then:

THEOREM 1. The functions $g$ and form a basis for the space of solutions of a second order homogeneous linear differential equation

$$
\Lambda=d^{2} / d x^{2}+P d / d x+Q, \quad P, Q \in K(X),
$$

on $X$. This equation will also have regular singular points.

Proof. Set

$$
P=-\frac{g d^{2} f / d x^{2}-f d^{2} g / d x^{2}}{g d f / d x-f d g / d x}, \quad Q=-\frac{(d f / d x) d^{2} g / d x^{2}-(d g / d x) d^{2} f / d x^{2}}{g d f / d x-f d g / d x} .
$$

Clearly both $P$ and $Q$ are single-valued and, moreover, because of the conditions at the cusps they are meromorphic on $X$. The regularity is also clear from the nature of the growth of $f$ and $g$ at the cusps.

Analogous classical results for elliptic modular functions can be found in Fricke and Klein [5], Bateman [1] and Ford [4]. Modern discussions of related differential operators are found in Katz [10], Serre [15], Weil [23] and Lang [12].

We remark that the choice of the parameter $x \in K(X)$ used in the derivation $d / d x$ is purely auxiliary. Moreover, by choosing $X_{0}$ small enough we can assume it is a good local parameter on all of $X_{0}$. We will somewhat abuse notation in using $x$ to denote both the parameter $x \in K(X)$ and a variable point $x \in X_{0}$.

The expression $W=g d f / d x-f d g / d x$ is known as the Wronskian of the differential equation. It is easily seen that $W=c \exp \left(-\int P d x\right)$ for some $c \in \mathbf{C}-\{0\}$ and, in our case, because $\Gamma \subset \mathrm{SL}_{2}(\mathbf{R})$ and because of the nature of the growth of $g$ and $f$ at the cusps, we have $W \in K(X)$.

Lastly, the representation

$$
\pi_{1}\left(X_{0}, x_{0}\right) \rightarrow \Gamma \subset \mathrm{SL}_{2}(\mathbf{R}),
$$

given by the analytic continuation of branches of $g$ and $f$ at $x_{0}$, is called the monodromy representation of $\Lambda$.

2. $K$-equations. Now let us assume $\Gamma \subset \mathrm{SL}_{2}(\mathbf{Z})$ and attempt to isolate the properties of this differential equation. We do this in some generality by defining the notion of $K$-equation on an arbitrary curve $X$.

Let $X$ be any complete smooth connected algebraic curve over $\mathbf{C}$ with function field denoted by $K(X)$. After fixing a parameter $x \in K(X)$, consider an algebraic differential equation on $X$

$$
\Lambda f=d^{2} f / d x^{2}+P d f / d x+Q f=0
$$

with $P$ and $Q$ in $K(X)$ and $f$ an unknown function. 
DEFinition 2. $\Lambda f=0$ is called a $K$-equation if it possesses two solutions $\omega_{1}$ and $\omega_{2}$, which are holomorphic nonvanishing multivalued functions on some Zariski open subset $X_{0}$ of $X$, satisfying:

(i) $\omega_{1}$ and $\omega_{2}$ form a basis of solutions,

(ii) for every closed path $\gamma \in \pi_{1}\left(X_{0}\right)$ the analytic continuation of $\left(\begin{array}{c}\omega_{1} \\ \omega_{2}\end{array}\right)$ around $\gamma$ is $M_{\gamma}\left(\begin{array}{c}\omega_{1} \\ \omega_{2}\end{array}\right)$ with $M_{\gamma} \in \mathrm{SL}_{2}(\mathbf{Z})$ (the monodromy representation), and

(iii) $\operatorname{Im}\left(\omega_{1} / \omega_{2}\right)>0$ on $X_{0}$ (positivity).

Such a pair of solutions is called a $K$-basis. In addition, since the monodromy is in $\mathrm{SL}_{2}(\mathbf{Z})$, the Wronskian $W=\exp \left(-\int P d x\right)$ is single-valued. As part of our definition we assume:

(iv) $W \in K(X)$.

This last condition is, in fact, equivalent to $\Lambda$ having regular singular points.

For example, when $\Gamma$ is contained in $\mathrm{SL}_{2}(\mathbf{Z})$ and $f$ is a classical automorphic form of weight one with respect to $\Gamma$, the differential equation determined by $f$ as in Theorem 1 is a $K$-equation with the pair $g, f$ serving as a $K$-basis.

The set of all $K$-equations on an arbitrary Riemann surface $X$ has been completely classified. We briefly recall that classification below.

Let $\Lambda f=0$ be a $K$-equation with $K$-basis $\omega_{1}$ and $\omega_{2}$. Consider the function $\mathscr{J}=J \circ \omega_{1} / \omega_{2}$,

$$
X_{0} \stackrel{\omega_{1} / \omega_{2}}{\rightarrow} \mathfrak{h} \stackrel{J}{\rightarrow} \mathbf{C}
$$

where $J$ is the elliptic modular function on the upper half-plane $\mathfrak{h}$. This $\mathscr{J}$ is a single-valued holomorphic function on $X_{0} \subset X$.

Proposition 3. $\mathscr{J} \in K(X)$.

Proof. This is an application of a result which appears in Kodaira [11] as Theorem 7.3.

We now determine all $K$-equations. Fix a $K$-equation $\Lambda f=0$ on $X$ with $K$-basis $\omega_{1}, \omega_{2}$ and let $\mathscr{J}=J\left(\omega_{1} / \omega_{2}\right)$. Say

$$
\Lambda f=d^{2} f / d x^{2}+P d f / d x+Q f=0 .
$$

THEOREM 4. There exists an algebraic function $\lambda$ on $X$ with $\lambda^{2} \in K(X)$ such that

$$
\begin{aligned}
P= & \frac{(d \mathscr{J} / d x)^{2}-\mathscr{J} d^{2} \mathscr{J} / d x^{2}}{\mathscr{J} d \mathscr{J} / d x}-\frac{d}{d x} \log \lambda^{2} \\
Q= & \frac{(d \mathscr{J} / d x)^{2}(31 \mathscr{J} / 144-1 / 36)}{\mathscr{J}^{2}(\mathscr{J}-1)^{2}}-\frac{(d \mathscr{J} / d x)^{2}-\mathscr{J} d^{2} \mathscr{J} / d x^{2}}{\mathscr{J} d \mathscr{J} / d x} \frac{d}{d x} \log \lambda \\
& -\frac{d^{2} \lambda / d x^{2}}{\lambda}+2\left[\frac{d \lambda / d x}{\lambda}\right]^{2} .
\end{aligned}
$$

This is known as the K-equation $\Lambda_{(\mathscr{J}, \lambda)}$.

Proof. See Stiller [19].

Note that in the case of the $K$-equation $\Lambda_{(\mathscr{J}, \lambda)}$ coming from an automorphic form $f$ of weight one with respect to $\Gamma \subset \mathrm{SL}_{2}(\mathbf{Z})$ the function $\mathscr{J}$ is none other than the elliptic modular function $J$. 
It is shown in Stiller [19] that $K$-equations are precisely those differential equations which arise as the Gauss-Manin connections associated to elliptic surfaces. To each elliptic surface over a curve $X$ we can associate a $K$-equation which is unique up to a simple transformation; conversely given a $K$-equation on $X$ we can associate to it an elliptic surface over $X$ unique up to generic isogeny. If, in addition, we specify a $K$-basis, then the surface will be uniquely determined. In the case of the $K$-equation coming from an automorphic form $f$ of weight one, we have a $K$-basis available, namely $g, f$. Thus there is a corresponding elliptic surface and we shall determine in the course of this paper explicitly which surface it is. In fact, the same surface arises for each choice of $f$ and the family of $K$-equations is just the family associated to that one elliptic surface as nmentioned above.

3. The associated elliptic surface. Let $\Gamma$ denote a subgroup of finite index in $\mathrm{SL}_{2}(\mathbf{Z})$ acting in the usual manner via linear fractional transformation on the upper half-plane $\mathfrak{h}$. We shall assume for simplicity that $\Gamma$ contains no torsion elements. This includes, for example, the case when $\Gamma=\Gamma(N)$ is the principal congruence subgroup of level $N, N \geqslant 3$, in $\mathrm{SL}_{2}(\mathbf{Z})$. The quotient $\mathfrak{h} / \Gamma=X_{0}$ is then a Zariski open subset of the compact Riemann surface $X$ gotten by removing the cusps. Moreover, the natural projection $\mathfrak{h} \rightarrow \mathfrak{h} / \Gamma=X_{0}$ serves to uniformize $X_{0}$, i.e. it is the universal covering and we can indentify $\pi_{1}\left(X_{0}\right)$ with $\Gamma$.

Our purpose in this section is to construct an elliptic surface $E$ over the compact curve $X$ and to study the Gauss-Manin connection (or Picard-Fuchs equation) of this family of elliptic curves.

For every element $\gamma \in \Gamma$ let

$$
f_{\gamma}(z)=\left(c_{\gamma} z+d_{\gamma}\right)^{-1} \quad \text { where } z \in \mathfrak{h}, \gamma=\left(\begin{array}{ll}
a_{\gamma} & b_{\gamma} \\
c_{\gamma} & d_{\gamma}
\end{array}\right)
$$

then we have $f_{\gamma \beta}(z)=f_{\gamma}(\beta z) f_{\beta}(z)$. Now, given a triple $\left(\gamma, n_{1}, n_{2}\right)$, with $\gamma \in \Gamma$ and $n_{1}, n_{2} \in \mathbf{Z}$, define an automorphism $g\left(\gamma, n_{1}, n_{2}\right)$ of $\mathfrak{h} \times \mathbf{C}$ by

$$
(z, t) \stackrel{g\left(\gamma, n_{1}, n_{2}\right)}{\rightarrow}\left(\gamma z, f_{\gamma}(z)\left(t+n_{1} z+n_{2}\right)\right) .
$$

We have

$$
\begin{aligned}
& g\left(\gamma, n_{1}, n_{2}\right) g\left(\beta, m_{1}, m_{2}\right)(z, t) \\
& \quad=g\left(\gamma \beta, a_{\beta} n_{1}+c_{\beta} n_{2}+m_{1}, b_{\beta} n_{1}+d_{\beta} n_{2}+m_{2}\right)(z, t),
\end{aligned}
$$

where

$$
\beta=\left(\begin{array}{ll}
a_{\beta} & b_{\beta} \\
c_{\beta} & d_{\beta}
\end{array}\right) .
$$

The energetic reader will verify that this set of automorphisms is actually a group $\mathscr{G}$ with this composition law. Its action is proper discontinuous fixed point free, so that the quotient $(\mathfrak{h} \times \mathbf{C}) / \mathscr{G}$ is a complex manifold which is clearly a family of elliptic curves over $\mathfrak{h} / \Gamma=X_{0}$ locally given by the lattice $z, 1$. This is essentially Kodaira's basic construction of an elliptic surface with a global section and prescribed functional and homological invariants (see Kodaira [11]). 
We can apply Kodaira's basic results to compactify this surface to get a proper smooth elliptic surface $E$ over the compact curve $X$ unique up to a biregular fiber-preserving map over $X$. The surface will have degenerate fibers of type $I_{b}\left(I_{b}^{*}\right)$, $b \geqslant 1$, over each regular (irregular) cusp where $\left(\begin{array}{ll}1 & b \\ 0 & 1\end{array}\right)\left(-\left(\begin{array}{ll}1 & b \\ 0 & 1\end{array}\right)\right)$ generates the isotropy group of the cusp after conjugating the cusp to " $\infty$ " in $\operatorname{SL}_{2}(\mathbf{Z})$. This surface will possess a section $s$

$$
E \underset{s}{\stackrel{\pi}{\leftrightarrows}} X
$$

(so that $\pi \circ s=1_{X}$, where $\pi$ is the projection of $E$ onto $X$ ) which makes the generic fiber $E^{\text {gen }}$ naturally an elliptic curve over the function field $K(X)$. Moreover, the functional invariant is just the elliptic modular function $J$ thought of as an element $\mathscr{J} \in K(X)$. The homological invariant can be viewed as the locally constant $\mathbf{Z} \oplus \mathbf{Z}$ sheaf on $X_{0}$ corresponding to the natural representation of $\pi_{1}\left(X_{0}, x_{0}\right)$ on $H^{1}\left(E_{x_{0}}, \mathbf{Z}\right)$, the integral cohomology of the fiber at some base point $x_{0} \in X_{0}$. The representation in this case is essentially the natural identification of $\pi_{1}\left(X_{0}, x_{0}\right)$ with $\Gamma \subset \mathrm{SL}_{2}(\mathbf{Z})$.

The surface above is called an elliptic modular surface and a full description can be found in Shioda [18]. We have repeated the construction so as to make clear some of the properties we shall be using later.

To the representation of $\pi_{1}\left(X_{0}, x_{0}\right) \rightarrow \Gamma \subset \mathrm{SL}_{2}(\mathbf{Z})$ we can associate an algebraic vector bundle on $X$ together with an integrable algebraic connection having regular singular points (Deligne [2], Griffiths [6]), which is known as the Gauss-Manin connection (Katz and Oda [9]). It can also be expressed as a second order algebraic differential equation

$$
\Lambda=d^{2} / d x^{2}+P d / d x+Q, \quad P, Q \in K(X),
$$

on $X$ having regular singular points. Using the results in Fricke and Klein [5] and Stiller [20], we have

THEOREM 5. For each choice of a Weierstrass model

$$
Y^{2}=4 X^{3}-g_{2} X-g_{3}, \quad g_{2}, g_{3} \in K(X),
$$

of the generic fiber of $E$ over $X$, the differential equation, which annihilates the periods of $d X / Y$ and expresses the Gauss-Manin connection, is the K-equation $\Lambda_{(\mathscr{J}, \lambda)}$, where

$$
\lambda^{-4} 27 \mathscr{J} /(\mathscr{J}-1)=g_{2} \text { and } \lambda^{-6} 27 \mathscr{J} /(\mathscr{J}-1)=g_{3} .
$$

Here $\mathscr{J}$ is the J-invariant of the elliptic curve and $\lambda^{2} \in K(X)$.

From this we see that is possible to select a $K$-basis $\omega_{1}, \omega_{2}$ so that under analytic continuation around $\rho \in \pi_{1}\left(X_{0}, x_{0}\right)$, corresponding to $\gamma \in \Gamma$, the monodromy matrix $M_{\rho}$ in $\mathrm{SL}_{2}(\mathbf{Z})$ is just $\gamma$. One should be cautious here and point out that the equation $\Lambda_{(\mathscr{g}, \lambda)}$ may have singularities on $X_{0}$. However, the only true singularities (i.e. where the local monodromy is not $\left(\begin{array}{ll}1 & 0 \\ 0 & 1\end{array}\right)$ ) will occur at the cusps. At singular points in $X_{0}$ the equation will possess local meromorphic solutions which will be single-valued. Such a point is called cosingular. 
THeOrem 6. Consider the natural projection $\mathfrak{h} \rightarrow \mathfrak{h} / \Gamma=X_{0}$ and lift $\omega_{2}(x)$ to a single-valued meromorphic function $\omega_{2}(z)$ on $\mathfrak{h}$. The function $\omega_{2}(z)$ is an automorphic form of weight one with respect to $\Gamma$. (For a classical special case of this result see Jordan [8].)

Proof. Let $\rho \in \pi_{1}\left(X_{0}, x_{0}\right)$ be a path corresponding to $\gamma=\left(\begin{array}{ll}a & b \\ c & d\end{array}\right) \in \Gamma$. Then under analytic continuation around $\rho$ we have

$$
\left(\begin{array}{l}
\omega_{1} \\
\omega_{2}
\end{array}\right) \rightarrow M_{\rho}\left(\begin{array}{l}
\omega_{1} \\
\omega_{2}
\end{array}\right)
$$

where $M_{\rho}$ is the monodromy matrix for the $K$-basis $\omega_{1}, \omega_{2}$ of $\Lambda_{(\mathscr{J}, \lambda)}$. Because we are working with the differential equation associated to an elliptic modular surface, we have

$$
M_{\rho}=\gamma=\left(\begin{array}{ll}
a & b \\
c & d
\end{array}\right) \in \Gamma .
$$

Moreover, $\omega_{1}(z) / \omega_{2}(z)=z$ because $\omega_{1}(z)$ and $\omega_{2}(z)$ are the periods for the lattice which gives the elliptic curve over each point in $X_{0}$ (minus, perhaps, a finite set where $\omega_{1}$ and $\omega_{2}$ are meromorphic) for the elliptic modular surface $E$ over $X$.

We see that $\omega_{2}(x)$ continues to $c \omega_{1}(x)+d \omega_{2}(x)$ around $\rho$ or

$$
\omega_{2}(\gamma z)=c \omega_{1}(z)+d \omega_{2}(z)=(c z+d) \omega_{2}(z) .
$$

Thus $\omega_{2}$ satisfies the correct transformation law and, finally, it will be meromorphic at the cusps because of the growth estimates on the solutions of equations with regular singularities.

So, given $\Gamma \subset \mathrm{SL}_{2}(\mathbf{Z})$ of finite index and no torsion, we can construct a canonical elliptic surface $E$ over $\mathfrak{h}^{*} / \Gamma=X$, called an elliptic modular surface, and by choosing a model for the generic fiber we can produce an automorphic form of weight one as a solution of the Picard-Fuchs equation for this surface.

Note that $\omega_{2}$ is uniquely picked out as a solution of $\Lambda_{(\mathscr{F}, \lambda)}$ up to a constant multiple. Namely, at the point $x \in X$ corresponding to the cusp at $\infty$, the $K$-equation $\Lambda_{(\mathscr{J}, \lambda)}$ will have exponents $(n, n)$ (or $(n / 2, n / 2)$ if $\infty$ is irregular) for some $n \in \mathbf{Z}$ (see Stiller [21]). This tells us that the global monodromy is conjugate in $\mathrm{GL}_{2}(\mathbf{C})$ to the local monodromy which is $\left(\begin{array}{ll}1 & 1 \\ 0 & 1\end{array}\right)$ (or $\left(\begin{array}{ll}-1 & -1 \\ 0 & -1\end{array}\right)$ in the irregular case), where locally a basis of solutions will be

$$
\begin{aligned}
& u_{2}=t^{n}(1+\cdots) \quad\left(\text { or } t^{n / 2}(1+\cdots)\right), \\
& u_{1}=u_{2}((1 / 2 \pi i) \log t+\text { holomorphic }),
\end{aligned}
$$

$t$ being a suitable local parameter. It follows immediately that $\omega_{2}$ is a multiple of $u_{2}$ since we wish to select $\omega_{1}, \omega_{2}$ so that the lifts to $\mathfrak{h}$ transform according to the homological invariant of $E$ over $X$. This fixes $\omega_{2}$ as some constant multiple of the invariant solution at the point $x$ corresponding to the cusp $\infty$ (and similarly for the irregular case).

Lastly, given one model

$$
Y^{2}=4 X^{3}-g_{2} X-g_{3}, \quad g_{2}, g_{3} \in K(X),
$$


for the generic fiber of $E$ over $X$, any other model is of the form

$$
Y^{2}=4 X^{3}-g^{-4} g_{2} X-g^{-6} g_{3}, \quad g \in K(X), g \neq 0 .
$$

It follows that if $\Lambda_{(\mathscr{J}, \lambda)}$ is the equation for the first model, then $\Lambda_{(\mathscr{J}, g \lambda)}$ is the equation for the second, and the basis $\omega_{1}, \omega_{2}$ in the first corresponds to $g \omega_{1}, g \omega_{2}$ in the second. In other words, the periods multiply and pointwise the lattices are homothetic except where $g$ has poles.

Of course when $g=c$ is constant, $\Lambda_{(\mathscr{J}, \lambda)}$ and $\Lambda_{(\mathscr{I}, c \lambda)}$ are equal. Thus, as we change the model we run through all nonzero multiples of $\omega_{2}$ by $g \in K(X)$ which means we get all automorphic forms of weight one.

In the last section we will explain how to choose $\lambda^{2} \in K(X)$ so that $\Lambda_{(\mathscr{J}, \lambda)}$ corresponds to the elliptic modular surface $E$ over $X$ for $\Gamma$. Remember that $\lambda$ is not unique as we can multiply by any nonzero modular function $g \in K(X)$. We will also explain how to tell if the resulting automorphic form is holomorphic or a cusp form and discuss computing $q$-expansions.

4. Forms of weight three and parabolic cohomology. Let $\Gamma$ be a subgroup of finite index in $\mathrm{SL}_{2}(\mathbf{Z})$ with no torsion and let $X$ be the compact Riemann surface $\mathfrak{h} * / \Gamma$. We will denote by $K(X)$ the function field of $X$ and by $E \stackrel{\pi}{\rightarrow} X$ the elliptic modular surface for $\Gamma$. We shall consider one of the second order linear homogeneous differential equations

$$
\Lambda=d^{2} / d x^{2}+P d / d x+Q, \quad P, Q \in K(X),
$$

which represents the Gauss-Manin connection for $E$ over $X$. As we have seen such an equation is a $K$-equation, i.e. $\Lambda=\Lambda_{(\mathscr{J}, \lambda)}$, where $\mathscr{J} \in K(X)$ corresponds to the elliptic modular function $J(z)$ on $\mathfrak{h}$ and $\lambda$ is an algebraic function whose square $\lambda^{2}$ is in $K(X)$. We denote by $X_{0}=\mathfrak{h} / \Gamma$ the Zariski open subset of $X$ obtained by deleting the cusps and select a base point $x_{0} \in X_{0}$ together with branches of $\omega_{1}$ and $\omega_{2}$ at $x_{0}$ as before.

Definition 7. A rational function $Z \in K(X)$ will be said to satisfy the parabolic residue condition for $\Lambda$ if the differential forms

$$
\left(-\omega_{2} Z / W\right) d x \quad \text { and } \quad\left(\omega_{1} Z / W\right) d x
$$

have zero residue at every point in $X_{0}$ (note that this is completely independent of the choice of basis $\omega_{1}, \omega_{2}$ because any solution of $\Lambda$ is a linear combination $a_{1} \omega_{1}+a_{2} \omega_{2}, a_{i} \in \mathbf{C}$, of $\omega_{1}, \omega_{2}$, and $\left(\left(a_{1} \omega_{1}+a_{2} \omega_{2}\right) Z / W\right) d x$ will also have zero residue at every point in $X_{0}$ ), and if at every regular cusp $s \in S=X-X_{0}$ (where the local monodromy is conjugate to $\left.\left(\begin{array}{ll}1 & n \\ 0 & 1\end{array}\right), n>0, n \in \mathbf{Z}\right)$ the differential form $\left(-\tilde{\omega}_{2} \mathrm{Z} / W\right) d x$ has zero residue, where $\tilde{\omega}_{2}$ is a local invariant solution at $s$. We denote by $L_{\Lambda}^{\text {para }}$ the subspace of $Z \in K(X)$ which satisfies the parabolic residue condition for $\Lambda$.

In what follows, we shall be interested in the inhomogeneous equations formed from the operator $\Lambda$ as given in (1) above. Thus, for $Z \in L_{\Lambda}^{\text {para }} \subset K(X)$ consider

$$
\Lambda f=Z, \quad f \text { unknown. }
$$


THEOREM 8. We have that

$$
f=\left(\int_{x_{0}}^{x} \frac{-\omega_{2} Z}{W} d x+c_{1}\right) \omega_{1}+\left(\int_{x_{0}}^{x} \frac{\omega_{1} Z}{W} d x+c_{2}\right) \omega_{2}
$$

as a multivalued meromorphic function on $X_{0}$. Here $c_{1}, c_{2} \in \mathbf{C}$ and $W=\omega_{1} d \omega_{2} / d x-$ $\omega_{2} d \omega_{1} / d x$ is the Wronskian of $\Lambda$. Note that we have somewhat abused notation in using $x$ to denote both the parameter $x \in K(X)$ and a variable point $x \in X_{0}$.

Proof. Apply variation of parameters to (2).

Note that the parameter $x$ need not be a good local parameter everywhere. However, if we replace the parameter $x \in K(X)$ by $t \in K(X)$ the new homogeneous equation is

$$
\Lambda^{\text {new }} f=\frac{d^{2} f}{d t^{2}}+\left(P \frac{d x}{d t}-\frac{d}{d t} \log \frac{d x}{d t}\right) \frac{d f}{d t}+Q\left(\frac{d x}{d t}\right)^{2} f=0,
$$

where $P$ and $Q$ are then original coefficients (see (1)). Moreover, we have for the inhomogeneous case

$$
\Lambda^{\text {new }} f=Z(d x / d t)^{2},
$$

where $f$ is the original inhomogeneous solution for (2) as in the theorem. Note that the Wronskian $W$, which clearly depends on a choice of derivation $d / d x$, becomes $W d x / d t$ when we change parameter. We can thus see directly from the formula in Theorem 8 that the expressions

$$
\left(-\omega_{2} Z / W\right) d x \text { and }\left(\omega_{1} Z / W\right) d x
$$

are naturally independent of parameter since

$$
\frac{-\omega_{2} Z(d x / d t)^{2}}{W(d x / d t)} d t=\frac{-\omega_{2} Z}{W} d x \text { and } \quad \frac{\omega_{1} Z(d x / d t)^{2}}{W(d x / d t)} d t=\frac{\omega_{1} Z}{W} d x
$$

We now return to consideration of the inhomogeneous equation.

Clearly we can continue the solution $f$ of the inhomogeneous equation in an unrestricted way throughout $X_{0}$. For $\rho \in \pi_{1}\left(X_{0}, x_{0}\right)$ corresponding to $\gamma \in \Gamma$ we have

$$
f \rightarrow f+m_{\gamma} \omega_{1}+n_{\gamma} \omega_{2}, \quad m_{\gamma}, n_{\gamma} \in \mathbf{C},
$$

because any two solutions of the inhomogeneous equation differ by a solution of the homogeneous equation.

Lifting $f$ to the upper half-plane viewed as the universal cover $\mathfrak{h}$ of $X_{0}=\mathfrak{h} / \Gamma$ we have a meromorphic single valued function (because of the residue condition) which we also denote by $f$ and which transforms as

$$
f(\gamma z)=f(z)+m_{\gamma} \omega_{1}(z)+n_{\gamma} \omega_{2}(z), \quad m_{\gamma}, n_{\gamma} \in \mathbf{C}, z \in \mathfrak{h}, \gamma \in \Gamma \subset \mathrm{SL}_{2}(\mathbf{Z}) .
$$

Note that $f$ satisfies growth conditions near the cusps similar to those for $\omega_{1}, \omega_{2}$ which are solutions of $\Lambda$, an equation with regular singular points.

As before we consider the inhomogeneous equation

$$
\Lambda f=Z, \quad Z \in L_{\Lambda}^{\text {para }} \subset K(X),
$$


and the solution as in Theorem 8

$$
f=\left(\int_{x_{0}}^{x} \frac{-\omega_{2} Z}{W} d x+c_{1}\right) \omega_{1}+\left(\int_{x_{0}}^{x} \frac{\omega_{1} Z}{W} d x+c_{2}\right) \omega_{2},
$$

where $x_{0} \in X_{0}$ is a base point and $\omega_{1}$ and $\omega_{2}$ are fixed branches at $x_{0}$. Because of our assumptions, $f$ is meromorphic multivalued on $X_{0}$ as is the function

$$
F=\frac{f}{\omega_{2}}=\left(\int_{x_{0}}^{x} \frac{-\omega_{2} Z}{W} d x+c_{1}\right) \omega+\left(\int_{x_{0}}^{x} \frac{\omega_{1} Z}{W} d x+c_{2}\right), \quad \omega=\frac{\omega_{1}}{\omega_{2}} .
$$

We define $F(z)$, a meromorphic single-valued function on the upper half-plane $\mathfrak{h}$, to be the lift of $F$ via the universal cover $\mathfrak{h} \rightarrow X_{0}=\mathfrak{h} / \Gamma$.

Note that for $\gamma \in \Gamma \subset \mathrm{SL}_{2}(\mathbf{Z})$ corresponding to $\rho \in \pi_{1}\left(X_{0}, x_{0}\right)$ we have

$$
F(\gamma z)=\left(c_{\gamma} z+d_{\gamma}\right)^{-1}\left(F(z)+m_{\gamma} z+n_{\gamma}\right), \quad z \in \mathfrak{h},
$$

where $m_{\gamma}, n_{\gamma} \in \mathbf{C}$ are called the periods of $F$ around $\gamma$ or $\rho$, and $\gamma=\left(\begin{array}{ll}a & b \\ c & d\end{array}\right) \in \Gamma \subset$ $\mathrm{SL}_{2}(\mathbf{Z})$ is the monodromy matrix for the continuation of $\omega_{1}, \omega_{2}$ around the corresponding path. Note that $\omega=\omega_{1} / \omega_{2}=z$ in this case. Again we remark that regularity imposes growth conditions on $F$ near the cusps.

As in Eichler [3], for each meromorphic $F$ on the upper half-plane $\mathfrak{h}$ satisfying (3), there is a meromorphic function $g$ on $\mathfrak{h}$ satisfying

$$
g(\gamma z)=\left(c_{\gamma} z+d_{\gamma}\right)^{3} g(z), \quad z \in \mathfrak{h}, \gamma \in \Gamma \subset \mathrm{SL}_{2}(\mathbf{Z}), \gamma=\left(\begin{array}{ll}
a_{\gamma} & b_{\gamma} \\
c_{\gamma} & d_{\gamma}
\end{array}\right),
$$

which is the monodromy as before. As such, $g$ is an automorphic form of weight three with respect to $\Gamma$. These functions are related by the formulas

$$
\begin{aligned}
& F(z)=\int_{z_{0}}^{z} g(t)(z-t) d t+c_{1} z+c_{2}, \\
& g(z)=\frac{1}{\pi i} \oint F(t)(t-z)^{-3} d t,
\end{aligned}
$$

where the path is taken around the boundary of a small disc about $z \in \mathfrak{h}$, and $z_{0} \in \mathfrak{h}$ lies over our base point $x_{0} \in X_{0}$.

LEMMA 9. $g(z)=\omega_{2}(z)^{3} Z(z) / W(z)^{2}$, all functions being understood as pull-backs via the canonical map $\mathfrak{h} \rightarrow X_{0}$ of the corresponding functions on $X_{0}$.

Proof. We analyze the situation locally near $x_{0}$. Here

$$
F(x)=\frac{f(x)}{\omega_{2}(x)}=\left(\int_{x_{0}}^{x} \frac{-\omega_{2} Z}{W} d x+c_{1}\right) \omega(x)+\left(\int_{x_{0}}^{x} \frac{\omega_{1} Z}{W} d x+c_{2}\right) .
$$

When we lift to the upper half-plane we get

$$
F(z)=\left(\int_{z_{0}}^{z} \frac{-\omega_{2}(z) Z(z)}{W(z)}\left(\frac{d x}{d z}\right) d z+c_{1}\right) z+\left(\int_{z_{0}}^{z} \frac{\omega_{1}(z) Z(z)}{W(z)}\left(\frac{d x}{d z}\right) d z+c_{2}\right) .
$$

Formula (5) is equivalent to

$$
g(z)=\left(d^{2} / d z^{2}\right) F(z),
$$


so we compute

$$
\begin{aligned}
\frac{d}{d z} F(z)= & \left(\int_{z_{0}}^{z} \frac{-\omega_{2}(z) Z(z)}{W(z)}\left(\frac{d x}{d z}\right) d z\right) \\
& +\frac{-\omega_{2}(z) Z(z)}{W(z)}\left(\frac{d z}{d x}\right)+\frac{\omega_{1}(z) Z(z)}{W(z)}\left(\frac{d z}{d x}\right) .
\end{aligned}
$$

But these last two terms add to zero as $\omega_{1} / \omega_{2}=z$. Thus

$$
g(z)=\frac{-\omega_{2}(z) Z(z)}{W(z)}\left(\frac{d x}{d z}\right)
$$

and, as $z=\omega(x)$, we have

$$
\frac{d z}{d x}=\frac{d}{d x} \omega(x)=\frac{d}{d x}\left(\frac{\omega_{1}(x)}{\omega_{2}(x)}\right)=\frac{-W(x)}{\omega_{2}(x)^{2}} .
$$

On $\mathfrak{h}$ this gives $g(z)=\omega_{2}(z)^{3} Z(z) / W(z)^{2}$.

Note that the automorphic form is completely independent of the choice of a particular solution to $\Lambda f=Z$, i.e. it is independent of $c_{1}, c_{2}$ as well as the base point $x_{0} \in X_{0}$. Moreover, it is independent of the parameter chosen since, as remarked above, $Z$ becomes $Z(d x / d t)^{2}$ and $W^{2}$ becomes $W^{2}(d x / d t)^{2}$.

Recall that on $\mathfrak{h}$ the function $f$ transforms as

$$
f(\gamma z)=f(z)+m_{\gamma} \omega_{1}(z)+n_{\gamma} \omega_{2}(z), \quad \gamma \in \Gamma, m_{\gamma}, n_{\gamma} \in \mathbf{C} .
$$

THEOREM 10.

$$
\begin{aligned}
{\left[m_{\gamma}, n_{\gamma}\right]=} & {\left[\int_{\rho} \frac{-\omega_{2} Z}{W} d x, \int_{\rho} \frac{\omega_{1} Z}{W} d x\right]\left(\begin{array}{ll}
a & b \\
c & d
\end{array}\right) } \\
& +\left[c_{1}, c_{2}\right]\left(\left(\begin{array}{ll}
a & b \\
c & d
\end{array}\right)-\left(\begin{array}{ll}
1 & 0 \\
0 & 1
\end{array}\right)\right),
\end{aligned}
$$

where $\rho \in \pi_{1}\left(X_{0}, x_{0}\right)$ corresponds to $\gamma=\left(\begin{array}{cc}a & b \\ c & d\end{array}\right) \in \Gamma \subset \mathrm{SL}_{2}(\mathbf{Z})$, which is the monodromy matrix for the continuation of $\omega_{1}, \omega_{2}$ around $\rho$. On the upper half-plane this becomes

$$
\begin{aligned}
{\left[m_{\gamma}, n_{\gamma}\right]=} & {\left[\int_{z_{0}}^{\gamma\left(z_{0}\right)} g(z) d z, \int_{z_{0}}^{\gamma\left(z_{0}\right)}-g(z) z d z\right]\left(\begin{array}{ll}
a & b \\
c & d
\end{array}\right) } \\
& +\left[c_{1}, c_{2}\right]\left(\left(\begin{array}{ll}
a & b \\
c & d
\end{array}\right)-\left(\begin{array}{ll}
1 & 0 \\
0 & 1
\end{array}\right)\right),
\end{aligned}
$$

and it follows that the assignment

$$
\begin{aligned}
\gamma & =\left(\begin{array}{ll}
a & b \\
c & d
\end{array}\right) \rightarrow\left(\begin{array}{ll}
a & b \\
c & d
\end{array}\right)\left(\begin{array}{cc}
0 & -1 \\
1 & 0
\end{array}\right)\left[m_{\gamma}, n_{\gamma}\right]^{t} \in \mathbf{C}^{2} \\
& =\left[\begin{array}{l}
\int_{z_{0}}^{\gamma\left(z_{0}\right)} g(z) z d z \\
\int_{z_{0}}^{\gamma\left(z_{0}\right)} g(z) d z
\end{array}\right]+\left[I-\left(\begin{array}{ll}
a & b \\
c & d
\end{array}\right)\right]\left[\begin{array}{c}
-c_{2} \\
c_{1}
\end{array}\right]
\end{aligned}
$$

is a group cocycle which is determined by $f$, or by $g$ up to coboundary. Note that all the integrals are well defined because $Z$ satisfies the residue conditions, i.e. $Z \in L^{\text {para }}$. 
Proof. Let $f$ be a solution to $\Lambda f=Z$ which by Theorem 8 has the form

$$
f=\left(\int_{x_{0}}^{x} \frac{-\omega_{2} Z}{W} d x+c_{1}\right) \omega_{1}+\left(\int_{x_{0}}^{x} \frac{\omega_{1} Z}{W} d x+c_{2}\right) \omega_{2} .
$$

Now under analytic (meromorphic) continuation around $\rho$ we have that

$$
\int_{x_{0}}^{x} \frac{-\omega_{2} Z}{W} d x \rightarrow \int_{x_{0}}^{x} \frac{-\left(c \omega_{1}+d \omega_{2}\right) Z}{W} d x+\int_{\rho} \frac{-\omega_{2} Z}{W} d x
$$

and likewise for $\int_{x_{0}}^{x} \omega_{1} Z / W d x$. An easy computation now gives that $f$ analytically continues to $f+m_{\gamma} \omega_{1}+n_{\gamma} \omega_{2}$ with $m_{\gamma}$ and $n_{\gamma}$ given by the stated formula. Lifting to the upper half-plane and using Lemma 9, which says that $g=\omega_{2}^{3} Z / W^{2}$, and the fact that $d z / d x=d \omega(x) / d x=-W / \omega_{2}^{2}$ gives the remainder of the result.

In fact, the periods $m_{\gamma}$ and $n_{\gamma}$ give a parabolic cocycle for $\Gamma$ (see Shimura [16]). In order to see this, we must show that for any parabolic element $\gamma=\left(\begin{array}{ll}a & b \\ c & d\end{array}\right) \in \Gamma$ there exists a vector $\left[\begin{array}{l}a_{1} \\ a_{2}\end{array}\right] \in \mathbf{C}^{2}$ such that

$$
\left(\begin{array}{ll}
a & b \\
c & d
\end{array}\right)\left(\begin{array}{cc}
0 & -1 \\
1 & 0
\end{array}\right)\left[m_{\gamma}, n_{\gamma}\right]^{t}=\left(\left(\begin{array}{ll}
1 & 0 \\
0 & 1
\end{array}\right)-\left(\begin{array}{ll}
a & b \\
c & d
\end{array}\right)\right)\left[\begin{array}{l}
a_{1} \\
a_{2}
\end{array}\right] .
$$

Without loss of generality, we can assume that the parabolic element $\gamma$ fixes the cusp $\infty$ and so takes the form

$$
\gamma=\left(\begin{array}{ll}
a & b \\
c & d
\end{array}\right)=\left(\begin{array}{ll}
1 & n \\
0 & 1
\end{array}\right), \quad n \in \mathbf{Z} .
$$

(In the case trace $\gamma=-2$ there is nothing to prove.) The path $\rho \in \pi_{1}\left(X_{0}, x_{0}\right)$ corresponding to $\gamma$ can be taken to go from $x_{0}$ to near the cusp, then to go around the cusp a number of times, and finally to return to $x_{0}$ along the original path. Calculating $\left[m_{\gamma}, n_{\gamma}\right]$ via Theorem 10 , we find that $m_{\gamma}=0$ because $\left(-\omega_{2} Z / W\right) d x$ is single-valued at the cusp and has zero residue when $Z \in L_{\Lambda}^{\text {para }}$. A simple calculation then shows that $a_{1}, a_{2}$ can be chosen to satisfy the desired condition.

Thus $Z \in L_{\Lambda}^{\text {para }}$ and the corresponding automorphic form $g$ determine a parabolic cohomology class, and this class arises naturally from the nature of the analytic continuation of the solutions to an inhomogeneous differential equation.

DEFINITION 11. Let $g$ be any meromorphic automorphic form of weight three with respect to $\Gamma \subset \mathrm{SL}_{2}(Z), \Gamma$ torsion-free of finite index. We say $g$ is of the second kind if $g=\omega_{2}^{3} Z / W^{2}$ for some $Z \in L_{\Lambda}^{\text {para }}$ and exact if $Z=\Lambda Z^{\prime}$ for some $Z^{\prime} \in K(X)$.

It is easy to see that any exact form is of the second kind and that $Z$ (of $f$ or $g$ ) gives the trivial parabolic cohomology class if and only if $Z=\Lambda Z^{\prime}$ for some $Z^{\prime} \in K(X)$ (i.e. $g$ is exact).

In a subsequent paper it will be shown that this correspondence induces bijections

$$
\frac{L_{\Lambda}^{\text {para }}}{\Lambda K(X)} \simeq \frac{\text { automorphic forms of second kind }}{\text { exact }} \simeq H_{p}^{1}\left(\Gamma, \mathbf{C}^{2}\right)
$$

with the parabolic cohomology group as in Shimura [16] and Eichler [3]. These bijections are closely related to, but by no means easily derived from, Shimura's isomorphism

$$
S_{3}(\Gamma) \simeq H_{p}^{1}\left(\Gamma, \mathbf{R}^{2}\right),
$$

where $S_{3}(\Gamma)$ is the space of usual cusp forms of weight three for $\Gamma$. 
Lastly, we remark that the periods, which are often of arithmetic significance (see Shimura [16]), can be computed by certain mixed abelian and hypergeometric integrals over a punctured sphere (see Stiller [22] for examples of such computations).

5. $q$-expansions and computations. In this final section we wish to show how one can use the differential equation to compute $q$-expansions, how to find the factor $\lambda$ appearing in the formula for the differential equation, and how to tell when the corresponding automorphic form of weight one is holomorphic or a cusp form.

Let $f$ be an automorphic form of weight one with respect to $\Gamma$ as before. We have seen that $f$ can be regarded as a multivalued solution of a $K$-equation $\Lambda_{(\mathscr{G}, \lambda)}$ on $X=\mathfrak{h} * / \Gamma$. In order to compute the $q$-expansion of $f$ at a cusp, we first shift the cusp to $\infty$ by a linear fractional transformation of the upper half-plane $\mathfrak{h}$. The effect of this is to conjugate $\Gamma$. Without any loss of generality, we thus assume that we are working with the cusp at $\infty$. Here $f$ corresponds to the invariant solution of $\Lambda_{(\mathscr{J}, \lambda)}$ up to a constant multiple (at least for a regular cusp). Since our equation has regular singular points we can apply well-known techniques to determine the solutions (see Ince [7], Griffiths [6], or Deligne [2]).

At this point, we need to calculate the local behavior of $\Lambda_{(\mathscr{J}, \lambda)}$ at a point in $X-X_{0}$. By Theorem 4

$$
\Lambda_{(\mathscr{J}, \lambda)} f=d^{2} f / d x^{2}+P d f / d x+Q f=0,
$$

where

$$
\begin{aligned}
P= & \frac{(d \mathscr{J} / d x)^{2}-\mathscr{J} d^{2} \mathscr{J} / d x^{2}}{\mathscr{J} d \mathscr{J} / d x}-\frac{d}{d x} \log \lambda^{2} \\
Q= & \frac{(d \mathscr{J} / d x)^{2}(31 \mathscr{J} / 144-1 / 36)}{\mathscr{J}^{2}(\mathscr{J}-1)^{2}}-\frac{(d \mathscr{J} / d x)^{2}-\mathscr{J} d^{2} \mathscr{J} / d x^{2}}{\mathscr{J} d \mathscr{J} / d x} \frac{d}{d x} \log \lambda \\
& -\frac{d^{2} \lambda / d x^{2}}{\lambda}+2\left(\frac{d \lambda / d x}{\lambda}\right)^{2} .
\end{aligned}
$$

Now the "parameter" $x$ does not provide a good local parameter everywhere and could therefore introduce singularities. This, however, is not the case; if $t$ is a local parameter at any point in $X$, then when we express $\Lambda_{(\mathscr{J}, \lambda)}$ locally it retains the above form with $t$ in place of $x$, i.e.

$$
\begin{aligned}
P= & \frac{(d \mathscr{J} / d t)^{2}-\mathscr{J} d^{2} \mathscr{J} / d t^{2}}{\mathscr{J} d \mathscr{J} / d t}-\frac{d}{d t} \log \lambda^{2} \\
Q= & \frac{(d \mathscr{J} / d t)^{2}(31 \mathscr{J} / 144-1 / 36)}{\mathscr{J}^{2}(\mathscr{J}-1)^{2}}-\frac{(d \mathscr{J} / d t)^{2}-\mathscr{J} d^{2} \mathscr{J} / d t^{2}}{\mathscr{J} d \mathscr{J} / d t} \frac{d}{d t} \log \lambda \\
& -\frac{d^{2} \lambda / d t^{2}}{\lambda}+2\left(\frac{d \lambda / d t}{\lambda}\right)^{2} .
\end{aligned}
$$

We see that the global "parameter" $x$ plays no role in determining the singularities. Thus the above form can be used in local calculations. In particular, we can take $t=q=e^{2 \pi i z / n}$ at the point corresponding to the cusp at $\infty$ where $\pm\left(\begin{array}{ll}1 & n \\ 0 & 1\end{array}\right) \in \Gamma$ generates the isotropy group at $\infty$. 
We will begin by calculating the local behavior of the $K$-equation $\Lambda_{(\mathscr{J}, 1)}$ (i.e. assume $\lambda=1$ ) when $\mathscr{J}$ has a pole of order $n \geqslant 1$. In terms of local coordinate $t$, $\mathscr{J}=c_{-n} / t^{n}+\cdots$. An easy calculation gives

$$
P=1 / t+\text { holomorphic, } \quad Q=0 / t^{2}+q_{-1} / t+\text { holomorphic. }
$$

The indicial equation is

$$
I(v)=v^{2}+\left(p_{-1}-1\right) v+q_{-2},
$$

where $P=p_{-1} / t+\cdots$ and $Q=q_{-2} / t^{2}+\cdots$. In this case $I(v)=v^{2}$ and the exponents are 0,0 . Thus the equation has a basis of solutions of the form

$$
u_{2}=1+\cdots, \quad u_{1}=u_{2}((1 / 2 \pi i) \log t+\text { holomorphic }),
$$

so that the local monodromy is $\left(\begin{array}{ll}1 & 1 \\ 0 & 1\end{array}\right)$. To reintroduce $\lambda$ is simple. $\Lambda_{(\mathscr{J}, \lambda)}$ will have solutions $\lambda u_{1}$ and $\lambda u_{2}$. Now $\lambda^{2} \in K(X)$, so if $\lambda^{2}=t^{r}$. (holomorphic nonvanishing), then the new exponents are $r / 2, r / 2$ and the local monodromy is $\pm\left(\begin{array}{ll}1 & 1 \\ 0 & 1\end{array}\right)$. Note that $u_{1}, u_{2}$ need not be a $K$-basis. Using Kodaira's classification for the monodromy at the singular fibers of an elliptic surface one can show that if $\omega_{1}, \omega_{2}$ is a $K$-basis, then after "shifting the cusp to $\infty$ " we have that the continuation of $\omega_{1}, \omega_{2}$ around the singularity gives $\pm\left(\begin{array}{ll}1 & n \\ 0 & 1\end{array}\right)$, where $n>0$ is the order of the pole of $\mathscr{J}$ - the negative case corresponding to an irregular cusp; the positive to a regular cusp.

Note that the elliptic modular surface defined in $\S 3$ for $\Gamma$ has good fibers at every point in $X_{0}=\mathfrak{h} / \Gamma$; thus the only other possible singularities of $\Lambda$ will be cosingular, i.e. the exponents must be distinct integers (see Stiller [21]) and the solutions will be meromorphic. Also because $\omega_{1} / \omega_{2}=z$ the order of $\omega_{2}$ (corresponding to a form $f$ of weight one) will equal the lower exponent and the difference between the exponents will be 1 at a cosingular point. We see that in order to compute the $q$-expansion up to a constant multiple we need to know the $q$-expansion of $J$, the elliptic modular function which is $\mathscr{J}$ in $\Lambda_{(\mathscr{J}, \lambda)}$, and the $q$-expansion for $\lambda^{2} \in K(X)$.

It is clear from the above discussion that if all the lower exponents are $\geqslant 0$ (including at the cosingular points), then the automorphic form $f$ will be holomorphic and if, in addition, the lower exponent (in fact, both exponents) are $\geqslant \frac{1}{2}$ at the cusps, the resulting form will be a cusp form. We state this as

THEOREM 12. If $f$ is an automorphic form of weight one coming from the $K$-equation $\Lambda_{(\mathscr{J}, \lambda)}$, then $f$ is holomorphic if and only if the lower exponent at each singularity of $\Lambda_{(\mathscr{J}, \lambda)}$ is $\geqslant 0$ and, in addition, $f$ will be a cusp form if the lower exponent at each cusp is $\geqslant \frac{1}{2}$.

We can also say when the inhomogeneous equations

$$
d^{2} f / d x^{2}+P d f / d x+Q f=\Lambda f=Z, \quad Z \in L_{\Lambda}^{\text {para }},
$$

give rise to cusp forms $g$ of weight three for $\Gamma$.

THEOREM 13. Let $\Gamma \subset \mathrm{SL}_{2}(\mathbf{Z})$ be a subgroup of finite index having no torsion and $\Lambda$ one of the second order differential equations representing the Gauss-Manin connection of the canonical elliptic modular surface $E$ over the modular curve $X=\mathfrak{h}^{*} / \Gamma$. An inhomogeneous equation

$$
\Lambda f=Z, \quad Z \in L_{\Lambda}^{\text {para }} \subset K(X),
$$


gives rise to a cusp of weight three for $\Gamma$ if and only if $Z \in L\left(\mathfrak{U}_{0}\right)=\{Z \in K(X)$ s.t. $\operatorname{div} Z+\mathfrak{A}>0\}$, where $\mathfrak{A}_{0}$ is the divisor

$$
\mathfrak{U}_{0}=\operatorname{div}(d x)^{2}+\sum_{\substack{x \in X \\ s_{x} \in \mathbf{Z}}}\left(-s_{x}+1\right)[x]+\sum_{\substack{x \in X \\ s_{x} \notin \mathbf{Z}}}\left(-s_{x}+\frac{3}{2}\right)[x],
$$

and $s_{x}$ is the largest root of the indicial equation for $\Lambda$ at $x$.

Proof. As we have seen above the two roots of the indicial equation for $\Lambda$ at $x \in X$ are integers except at the irregular cusps where they are half-integers. Since for most points the roots are 0,1 , we have $s_{x}=1$ for all but finitely many points and the divisor $\mathfrak{U}_{0}$ is well defined.

To see that the automorphic form associated to

$$
\Lambda f=Z, \quad Z \in L\left(\mathfrak{A}_{0}\right),
$$

is a cusp form is purely a local calculation on $X$. For example, if $t$ is a local parameter at $x \in X$ and $x$ is a regular cusp, then in terms of $t$ we have $\Lambda f=$ $Z(d x / d t)^{2}$, with

$$
\begin{aligned}
& \omega_{2}=t^{s_{x}} \cdot(\text { holomorphic nonvanishing }), \\
& W=t^{2 s_{x}-1} \cdot(\text { holomorphic nonvanishing). }
\end{aligned}
$$

As $g$ is the pull-back of $\omega_{2}^{3} Z(d x / d t)^{2} / W^{2}$ we see that it will vanish at the cusp if and only if ord $_{x}\left(Z(d x / d t)^{2}\right) \geqslant s_{x}-1$. The same analysis at the irregular cusps and cosingular points gives the result.

We remark that it follows easily from Shimura [16] or Shioda [18] that no nonzero $Z \in L\left(\mathfrak{U}_{0}\right)$ corresponds to an exact form $g$, and hence the map $S_{3}(\Gamma) \rightarrow H_{p}^{1}\left(\Gamma, \mathbf{C}^{2}\right)$ is injective.

Lastly we wish to make some remarks on choosing $\lambda^{2} \in K(X)$ when $\Gamma$ is a principal congruence subgroup $\Gamma(N), N \geqslant 3$. If one examines all the singularities of the equation $\Lambda_{(\mathscr{J}, 1)}$ with $\mathscr{J}$ the elliptic modular function $J$, one sees that it has the following singularities:

(1) At the cusps the exponents are $(0,0)$.

(2) At the $\mu / 3$ points where $J=0$ the exponents are $-\frac{1}{2}, \frac{1}{2}$. (Here $\mu$ is the index of $\Gamma \cdot\{ \pm I\}$ in $\mathrm{SL}_{2}(\mathbf{Z})$.)

(3) At the $\mu / 2$ points where $J=1$ the exponents are $\frac{1}{2}, \frac{3}{2}$.

(4) At all other points $\Lambda$ is holomorphic and the exponents are 0,1 .

Thus we must choose $\lambda^{2}$ to have odd order at each of the $5 \mu / 6$ points in cases (2) and (3), and to have even order elsewhere. Once one $\lambda$ is found all others are of the form $g \lambda$ with $g \in K(X)$.

Now when $\Gamma=\Gamma(N)$ with $N \equiv 0 \bmod 4$ we can actually find $\lambda$. Note that when $N$ is even, $\Gamma(N) \subset \Gamma(2)$ and the Legendre $\lambda$-function (which we denote by $L$ to avoid confusion with $\lambda$ above) is a modular function for $\Gamma(N)$. Recall (Robert [14]) that

$$
J=\frac{4}{27} \frac{\left(1-L+L^{2}\right)^{3}}{L^{2}(1-L)^{2}}
$$


and we have the maps

$$
\mathfrak{h}^{*} / \Gamma(N) \stackrel{\alpha}{\rightarrow} \mathfrak{h} * / \Gamma(2) \stackrel{\beta}{\rightarrow} \mathfrak{h}^{*} / \mathrm{SL}_{2}(\mathbf{Z}),
$$

where $\alpha$ is unramified except over the cusps and $\beta$ is ramified to order 2 at $0,1, \infty$ over $\infty$, to order 3 at $e^{2 \pi i / 6}, e^{-2 \pi i / 6}$ over 0 , and to order 2 at $-1,2, \frac{1}{2}$ over 1 .

If we let

$$
\begin{aligned}
\lambda^{2} & =\frac{\left(\alpha^{*}(L)-e^{2 \pi i / 6}\right)\left(\alpha^{*}(L)-e^{-2 \pi i / 6}\right)}{\left(\alpha^{*}(L)+1\right)\left(\alpha^{*}(L)-2\right)\left(\alpha^{*}(L)-1 / 2\right)} \\
& =\alpha^{*}\left(\frac{L^{2}-L+1}{(L+1)(L+2)(L-1 / 2)}\right),
\end{aligned}
$$

where $\alpha^{*}$ means view $L$ (or whatever) as a modular function for $\Gamma(N)$, then the equation $\Lambda_{(\mathscr{J}, \lambda)}$ will be holomorphic on $\mathfrak{h} / \Gamma(N)=X_{0}$, so the exponents will be 0,1 .

In order to determine the behavior at the cusps, observe that $\Gamma(N)$ has $\mu / N$ cusps and that $\alpha$ maps $\mu / 3 N$ of them to each of the points $0,1, \infty \in \mathfrak{h} * / \Gamma(2)$ with ramification $N / 2$. At the $2 \mu / 3 N$ cusps over 0,1 the function $\lambda^{2}$ is holomorphic nonvanishing, so $\Lambda_{(\mathscr{g}, \lambda)}$ will have exponents $(0,0)$, but at each of the $\mu / 3 N$ cusps over $\infty$ the function $\lambda^{2}$ has a zero of order $N / 2$ so that $\Lambda_{(\mathscr{g}, \lambda)}$ will have exponents $(N / 4, N / 4)$.

Thus if $N \equiv 0 \bmod 4$ the function $\lambda^{2}$ above will serve to produce one of the desired $K$-equations. This equation then determines (up to a constant multiple) an automorphic form $f$ of weight one with respect to $\Gamma(N)$. Moreover, $f$ will be holomorphic nonvanishing on $\mathfrak{h}$, will vanish to order $N / 4$ at $\mu / 3 N$ cusps, and will be holomorphic nonvanishing at the $2 \mu / 3 N$ remaining cusps. One can easily compute its $q$-expansion in terms of the $q$-expansions of the elliptic modular function $J$ and the Legendre $\lambda$-function which we have denoted by $L$. In addition, it is easy to see that $f$ can be normalized to have Fourier coefficients in $\mathbf{Q}$ since both $J$ and $L$ have rational Fourier coefficients.

For example, for $\Gamma=\Gamma(4)$ the principal congruence subgroup of level four, the differential equation above is

$$
\Lambda_{\Gamma(4)}=\frac{d^{2}}{d x^{2}}+\frac{x^{4}-4 x^{2}-1}{x\left(x^{2}-1\right)\left(x^{2}+1\right)} \frac{d}{d x}+\frac{4}{\left(x^{2}-1\right)^{2}},
$$

where $X=\mathfrak{h}^{*} / \Gamma(4)$ is identified with the Riemann $x$-sphere by choosing a modular function $x(z)$ which satisfies

$$
L(z)=\left(\frac{x(z)^{2}+1}{x(z)^{2}-1}\right)^{2}
$$

suitably normalized (see Petersson [13] and Stiller [22]). The map

$$
\frac{\mathfrak{h}^{*}}{\Gamma(4)} \rightarrow \frac{\mathfrak{h}^{*}}{\Gamma(2)}, \quad x \rightarrow\left(\frac{x^{2}+1}{x^{2}-1}\right)^{2}=L
$$

shows that $\pm i$ go to $0, \pm 1$ go to $\infty$, and $0, \infty$ go to 1 , and as is easily checked the 
exponents are $(0,0)$ at $i, 0, \infty$ and $(1,1)$ at \pm 1 as observed above. The $q$-expansion of the associated normalized holomorphic form of weight one at $i \infty$ (which corresponds to $i$ on the $x$-sphere) can then be calculated (see Stiller [22]).

\section{REFERENCES}

1. Bateman Manuscript Project, Higher transcendental functions, Vol. 3, McGraw-Hill, New York, 1953, pp. 20-23.

2. P. Deligne, Equations différentielles à points singuliers réguliers, Lecture Notes in Math., vol. 163, Springer-Verlag, Berlin, Heidelberg and New York, 1970.

3. M. Eichler, Quadratische Formen und Modulfunktionen, Acta Arith. 4 (1958), 217-239.

4. L. R. Ford, A utomorphic functions, McGraw-Hill, New York, 1929, p. 99.

5. R. Fricke and F. Klein, Vorlesung über die Theorie der elliptischen Modulfunktionen, Teubner, Leipzig, 1890.

6. P. Griffiths, Differential equations on algebraic varieties, Princeton lectures, unpublished.

7. E. Ince, Ordinary differential equations, Dover, New York, 1956.

8. C. Jordan, Cours d'analyse, Gauthier-Villars, Paris, 1909.

9. N. Katz and T. Oda, On the differentiation of De Rham cohomology classes with respect to parameters, J. Math. Kyoto Univ. 8 (1968), 199-213.

10. N. Katz, P-adic properties of modular schemes and modular forms, Lecture Notes in Math., vol. 350, Springer-Verlag, Berlin, 1973, pp. 168-171.

11. K. Kodaira, On compact analytic surfaces. II, Ann. of Math. (2) 77 (1963), 563-626.

12. S. Lang, Introduction to modular forms, Springer-Verlag, Berlin, 1976, p. 161.

13. H. Petersson, Über die Kongruenzgrupen der Strufe 4, J. Reine Angew. Math. 212 (1963), 64-72.

14. A. Robert, Elliptic curves, Lecture Notes in Math., vol. 326, Springer-Verlag, Berlin, Heidleberg and New York, 1973.

15. J.-P. Serre, Congruences et formes modulaires, Exposé 416, Séminaire N. Bourbaki, 1971/72, Lecture Notes in Math., vol. 317, Springer-Verlag, Berlin, Heidleberg and New York, 1973.

16. G. Shimura, Sur les intégrales attachées aux formes automorphes, J. Math. Soc. Japan 11 (1959).

17. , Introduction to the arithmetic theory of automorphic forms, Princeton Univ. Press, Princeton, N. J., 1971.

18. T. Shioda, On elliptic modular surfaces, J. Math. Soc. Japan 24 (1972), 20-59.

19. P. Stiller, Differential equations associated with elliptic surfaces, J. Math. Soc. Japan 32 (1981).

20. Elliptic curves over function fields and the Picard number, Amer. J. Math. 102 (1980), 565-593.

21. Automorphic forms and the Picard number of an elliptic surface, Aspects of Math. E, Vol. E5, Vieweg, Braunschweig, 1984.

22. Special values of Dirichlet series, monodromy, and the periods of automorphic forms, Mem. Amer. Math. Soc. No. 299 (1984).

23. A. Weil, Elliptic functions according to Eisenstein and Kronecker, Springer-Verlag, New York, 1976.

SONDERForschungsbereich, Universität Bonn, Bonn, Federal RePUblic of Germany

Department of Mathematics, Texas A \& M University, College Station, Texas 77843 (Current address) 